\title{
Pathogenesis of Infection with 2009 Pandemic H1N1 Influenza Virus in Isogenic Guinea Pigs after Intranasal or Intratracheal Inoculation
}

\author{
Lidewij C.M. Wiersma, ${ }^{*}$ Stella E. Vogelzang-van Trierum, ${ }^{*}$ Geert van Amerongen, ${ }^{* \dagger}$ Peter van Run, ${ }^{*}$ Nella J. Nieuwkoop, ${ }^{*}$
} Mechtild Ladwig, ${ }^{\ddagger}$ Stefanie Banneke, ${ }^{\ddagger}$ Hubert Schaefer, ${ }^{\S}$ Thijs Kuiken, ${ }^{*}$ Ron A.M. Fouchier, ${ }^{*}$ Albert D.M.E. Osterhaus, ${ }^{* \dagger}$ and Guus F. Rimmelzwaan* ${ }^{\dagger}$

From the Viroscience Laboratory, ${ }^{*}$ Erasmus Medical Centre, Rotterdam, the Netherlands; Viroclinics Biosciences BV ${ }^{\dagger}$ Rotterdam, the Netherlands; Department of Experimental Toxicology and Centre for Documentation and Evaluation of Alternatives to Animal Experiments, ${ }^{\ddagger}$ the Federal Institute for Risk Assessment, Berlin, Germany; and Experimental Immunology, ${ }^{\S}$ the Robert Koch Institute, Berlin, Germany

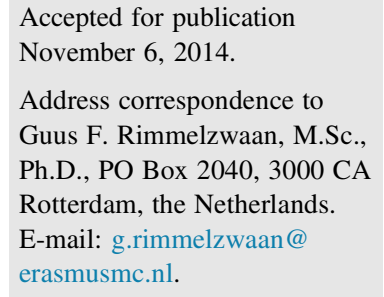

\begin{abstract}
To elucidate the pathogenesis and transmission of influenza virus, the ferret model is typically used. To investigate protective immune responses, the use of inbred mouse strains has proven invaluable. Here, we describe a study with isogenic guinea pigs, which would uniquely combine the advantages of the mouse and ferret models for influenza virus infection. Strain 2 isogenic guinea pigs were inoculated with H1N1pdm09 influenza virus $\mathrm{A} /$ Netherlands $/ 602 / 09$ by the intranasal or intratracheal route. Viral replication kinetics were assessed by determining virus titers in nasal swabs and respiratory tissues, which were also used to assess histopathologic changes and the number of infected cells. In all guinea pigs, virus titers peaked in nasal secretions at day 2 after inoculation. Intranasal inoculation resulted in higher virus excretion via the nose and higher virus titers in the nasal turbinates than intratracheal inoculation. After intranasal inoculation, infectious virus was recovered only from nasal epithelium; after intratracheal inoculation, it was recovered also from trachea, lung, and cerebrum. Histopathologic changes corresponded with virus antigen distribution, being largely limited to nasal epithelium for intranasally infected guinea pigs and more widespread in the respiratory tract for intratracheally infected guinea pigs. In summary, isogenic guinea pigs show promise as a model to investigate the role of humoral and cell-mediated immunities to influenza and their effect on virus transmission. (Am J Pathol 2015, 185: 643-650; http://dx.doi.org/10.1016/j.ajpath.2014.11.012)
\end{abstract}

Influenza viruses are an important cause of respiratory tract infections in humans. Seasonal influenza A/H1N1, A/H3N2, and B-viruses cause epidemics in the human population annually [WHO: Influenza (seasonal) fact sheet number 211. Edited 2014, available at http://www.who.int/mediacentrel factsheets/fs211/en]. Occasionally, novel antigenically distinct influenza viruses cause pandemic outbreaks with increased rates of morbidity and mortality. The last pandemic occurred in 2009 and was caused by A/H1N1 viruses of swine origin., Furthermore, zoonotic transmissions of avian influenza virus of various subtypes (eg, H7N9 and H5N1) are reported and may cause relatively large numbers of fatalities. $^{3-5}$

To obtain understanding of these viruses for pathogenesis, virulence, transmissibility, and immunity, various animal models are being used. ${ }^{6-9}$ Commonly, infection of ferrets is considered the preferred animal model for human influenza virus infections, because the pathogenesis in ferrets most closely resembles that observed in humans. ${ }^{8,10}$ In addition, ferrets and humans show similarities in receptor distribution in the respiratory tract, thus allowing for virus replication in the upper and/or lower respiratory tract, depending on the origin of the viruses tested and the route of inoculation. ${ }^{11,12}$ Because virus replication in the ferret upper respiratory tract is considered indicative for transmissibility of influenza viruses between humans, ferrets are also used for

Supported by EU-FP7 FLUPIG grant FP7-GA258084 (G.F.R. and L.C.M.W.).

Disclosures: G.v.A., A.D.M.E.O., and G.F.R. are employees of Viroclinics Biosciences. 
transmission studies. ${ }^{7,8}$ Other species that may be used to study transmission include (outbred) guinea pigs ${ }^{7,13}$ and, as recently identified, the common marmoset. ${ }^{14}$

The animal models mentioned in the paragraph above each have their advantages and limitations. It has proven difficult to correlate markers of cellular immunity with pathogenesis and transmission in these animal species.

For investigating (cellular) immunity to influenza virus infections inbred mouse strains are commonly used, in part because many reagents are available for the identification and isolation of cells of the murine immune system. These models have provided a wealth of information on immune function. ${ }^{15}$ The use of inbred animals also allows performing adoptive transfer experiments with isolated T- or B-lymphocyte populations or other cells of the immune system to assess their contribution to protective immunity. ${ }^{16}$ However, mice transmit influenza viruses inefficiently, and it is doubtful whether the pathogenesis of infection resembles that of humans.?

The choice of animal model for any given study should therefore be selected on the basis of the research question. Ideally, an animal model is used that combines the advantages of the ferret and the mouse model. Therefore, we were interested in using inbred guinea pigs (Cavia porcellus). We used strain 2 guinea pigs; this designation follows the principles devised by geneticist Sewall Wright, a pioneer in animal (specifically guinea pig) inheritance patterns. Currently, the only inbred strains still available are strain 2 and strain 13. It is already demonstrated that transfer of lymphocyte populations is possible in these guinea pigs. ${ }^{17,18}$ In addition, sufficient immunologic reagents are available to identify and isolate lymphocyte subsets. ${ }^{19}$ Other advantages of the use of (isogenic) guinea pigs is that they are susceptible to both unadapted avian and human influenza viruses and can be used to investigate transmissibility of these viruses in relation to the presence of (cellular) immunity. ${ }^{7}$

Typically, guinea pigs are inoculated with influenza virus by the intranasal (i.n.) route, ${ }^{7,8}$ presumably because of the ease of administration. Intratracheal (i.t.) inoculation through intubation is described ${ }^{20}$ but is challenging because the diameter of the trachea is small and the cervicocranial anatomy of the guinea pig precludes simple visualization of the larynx via the mouth. To overcome these obstacles, inoculation with virus by the i.t. route can be performed transcutaneously (surgically), which was used previously with inactivated influenza virus ${ }^{21}$ and other pathogens. ${ }^{22}$

Our aim was to investigate the pathogenesis of influenza virus infection in inbred strain 2 guinea pigs and to compare different routes of inoculation, because it was shown in ferrets that the route of inoculation has a profound effect on the pathogenesis. ${ }^{12}$

For infection of isogenic strain 2 guinea pigs, we used 2009 pandemic $\mathrm{A} / \mathrm{H} 1 \mathrm{~N} 1$ influenza virus, which has continued to circulate as a seasonal virus since 2009 .

Although studies were conducted into its transmission, ${ }^{23}$ knowledge about pathogenesis and virus replication kinetics of this virus strain in particular, and influenza viruses in general, is still limited in guinea pigs. ${ }^{24}$
To pave the way for new lines of research into viral transmission and cellular immunity, this study was performed to assess replication kinetics and pathogenesis of influenza A (H1N1) pdm09 virus infection via i.n. or i.t. inoculation in strain 2 isogenic guinea pigs.

\section{Materials and Methods}

\section{Animals and Experimental Design}

All experiments were performed in accordance with an animal experimentation protocol approved by an independent Animal Welfare Committee (DEC Consult) and in compliance with national and European legislation. Twenty-two female, 12- to 16-week-old ( 320 to $520 \mathrm{~g}$ ) isogenic strain 2 guinea pigs were purposely bred at the Federal Institute for Risk Assessment in Berlin, Germany. Before the start of the experiment, guinea pigs were tested for the presence of serum antibodies to circulating influenza viruses $\mathrm{A} / \mathrm{H} 1 \mathrm{~N} 1, \mathrm{~A} / \mathrm{H} 3 \mathrm{~N} 2$, and B/Yamagata-like by using epidemic and corresponding vaccine strains with the hemagglutination inhibition assay, as described (WHO Manual on Animal Influenza Diagnosis and Surveillance. Edited 2002, available at $h t t p: / / w w w . w h o . i n t / c s r /$ resources/publications/influenza/en/whocdscsrncs20025rev. $p d f)$. Influenza virus-specific antibodies were not detected in any of the guinea pigs.

Guinea pigs were housed in groups of four or six per negatively pressurized BSL3 isolator unit at the animal facilities of the Erasmus Medical Center, on a 12-hour light/dark cycle and with ad libitum access to food and water.

Twelve guinea pigs were inoculated by the i.n. route under ketamine and medetomidine anesthesia by instilling $150 \mu \mathrm{L}$ of virus per nostril [total volume $300 \mu \mathrm{L}$ per guinea pig, containing $2 \times 10^{6}$ median tissue culture infectious dose $\left(\mathrm{TCID}_{50}\right)$ of influenza virus A/Netherlands/602/2009] with the animal in an upright position. The anesthetic was antagonized with atipamezole, and, during recovery of the guinea pigs, the head and nose were kept slightly elevated with respect to the rump to prevent the inoculum from flowing out of the nostrils. Nasal, pharyngeal, and rectal swabs were taken daily, and guinea pigs were weighed before inoculation on day 0 and on days $1,2,3,4(n=12)$, and $7(n=6)$ after inoculation under isofluorane anesthesia (4\% induction, $2 \%$ maintenance). On day 4 and 7 , six guinea pigs were sacrificed by exsanguination under anesthesia with ketamine and medetomidine, and autopsy was performed.

Ten guinea pigs were inoculated via the i.t. route. To this end, guinea pigs were anesthetized with ketamine and medetomidine, and $500 \mu \mathrm{L}$ of phosphate-buffered saline that contained $2 \times 10^{6}$ TCID $_{50}$ of virus was injected transcutaneously (surgically) directly into the trachea, while the animal was in dorsal recumbency. The anesthetic was antagonized with atipamezole, and during recovery guinea pigs were again positioned with their heads slightly elevated with respect to the rump to prevent the inoculum from flowing out via either the pharyngeal or nasal cavity. Nasal, 
pharyngeal, and rectal swabs were taken daily. Nasal swabbing was performed by inserting a thin (urethral) swab carefully into the nasal vestibule. Rectal and pharyngeal swabbing was performed by inserting the swab approximately $3 \mathrm{~cm}$ until slight resistance was felt; the swab was carefully twisted several times and retracted. Guinea pigs were weighed and swabbed before inoculation on day 0 and on days $1(n=10), 2(n=8), 3(n=6), 4(n=4)$, and 7 $(n=2)$ after inoculation under isofluorane anesthesia. On day $1,2,3,4$, and 7 after inoculation, two guinea pigs were sacrificed per time point by exsanguination as described earlier, and autopsies were performed.

\section{Virus Preparation}

Influenza virus A/Netherlands/602/2009 (A(H1N1)pdm09) was propagated in confluent Madin-Darby canine kidney cells. Culture supernatant fluids were harvested after the appearance of cytopathic changes, cleared by low-speed centrifugation, and stored at $-80^{\circ} \mathrm{C}$. The virus titer was determined in Madin-Darby canine kidney cells as described previously. ${ }^{25}$ Virus was diluted in phosphatebuffered saline to the desired concentration of $2 \times 10^{6}$ $\mathrm{TCID}_{50}$ per $300 \mu \mathrm{L}$ for i.n. and $500 \mu \mathrm{L}$ for i.t. inoculations.

\section{Pathologic Examination and Immunohistochemistry}

Autopsies were performed according to standard procedures. The trachea was clamped before opening the thorax, and inflated lungs were visually inspected to assess the percentage of pulmonary tissue affected. Samples of nasal turbinate, nasal septum, trachea, tracheobronchial lymph node, bronchus, lung, cerebrum (including the olfactory bulb), cerebellum, heart, liver, kidney, spleen, duodenum, and colon were fixed in 10\% neutral-buffered formalin. For improved histologic assessment, the entire right lung was inflated with formalin before fixation. Formalin-fixed tissues were embedded in paraffin, sectioned at $4 \mu \mathrm{m}$, and stained with hematoxylin and eosin for histologic evaluation. For immunohistochemical (IHC) evaluation, antigen was retrieved with $0.1 \%$ protease in phosphate-buffered saline at $37^{\circ} \mathrm{C}$ for 10 minutes, and tissues were stained with an immunoperoxidase method by using a monoclonal antibody against influenza nucleoprotein (to assess influenza antigen distribution) or against major basic protein antibody, clone BMK-13 (for identification of eosinophils; AbD Serotec, Raleigh, NC). For IHC analysis, IgG2a isotype controls of each tissue and a positive control (for influenza) were included. All slides were evaluated by light microscopy without knowledge of the identity of the guinea pigs. Cranial and caudal lung lobes were sectioned both longitudinally and cross-sectionally, and hematoxylin and eosin-stained slides were semiquantitatively assessed with previously described criteria. ${ }^{26}$
Influenza antigen IHC analysis was semiquantitatively evaluated without knowledge of the identity of the guinea pigs by visually estimating the number of infected cells.

\section{Assessment of Virus Titers in Tissues and Swabs}

Nasal, pharyngeal, and rectal swabs were stored at $-70^{\circ} \mathrm{C}$ in transport medium (Hanks' balanced salt solution with $0.5 \%$ lactalbumin, $10 \%$ glycerol, $200 \mathrm{U} / \mathrm{mL}$ penicillin, $200 \mu \mathrm{g} / \mathrm{mL}$ streptomycin, $100 \mathrm{U} / \mathrm{mL}$ polymyxin B sulfate, $250 \mu \mathrm{g} / \mathrm{mL}$ gentamicin, and $50 \mathrm{U} / \mathrm{mL}$ nystatin; ICN Pharmaceuticals, Zoetermeer, the Netherlands). Samples of nasal turbinates, trachea, lung, cerebrum (including olfactory bulb), cerebellum, liver, spleen, lung, duodenum, and colon were stored at $-70^{\circ} \mathrm{C}$. For determination of viral titers, tissues were weighed and homogenized (FastPrep-24 homogenizer; MP Biomedicals, Eindhoven, the Netherlands) in transport medium and cleared of tissue debris by centrifugation ( 1 minute at 10,000 rpm). Tenfold serial dilutions of swabs and processed tissue samples were used to infect Madin-Darby canine kidney cells in quadruplicate. On day 5 after infection of cells, hemagglutination activity of the culture supernatant fluids was assessed to demonstrate the presence of progeny virus. ${ }^{25}$ Mean TCID $_{50}$ (expressed per milliliter for swabs and per gram for tissue samples) was calculated with the Spearman-Karber standard equation.

\section{Results}

\section{Clinical Signs}

Guinea pigs displayed few clinical signs. Two days after inoculation, recovery from isofluorane anesthesia was slower for all guinea pigs, and guinea pigs in the i.t.-inoculated

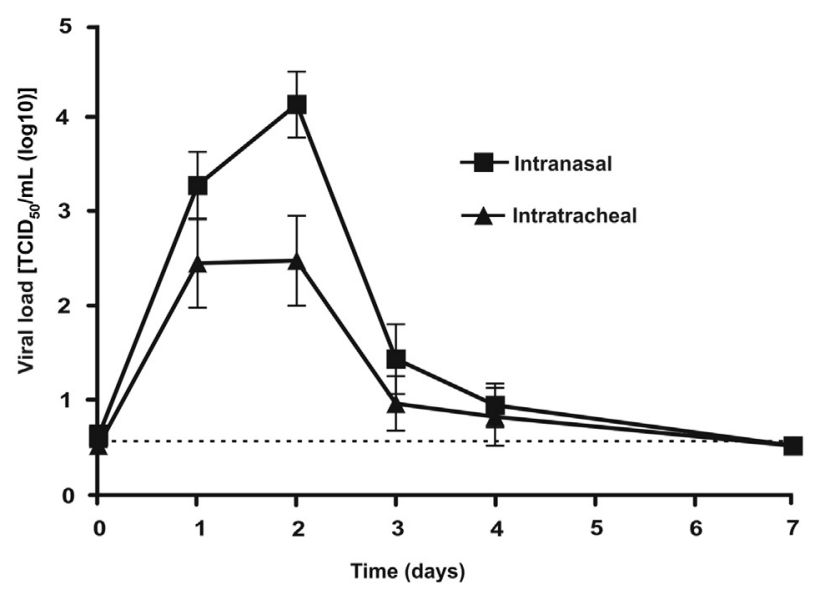

Figure 1 Viral load in nasal swabs after intranasal or intratracheal inoculation with influenza $\mathrm{A}(\mathrm{H} 1 \mathrm{~N} 1) \mathrm{pdm} 09$ virus. Dashed line indicates limit of detection $<0.75 \mathrm{TCID}_{50} / \mathrm{mL}$ (10log). Intranasal: $n=12$ for day 0 to $4, n=6$ for day 7 after inoculation. Intratracheal: $n=10$ for day 0 to $1, n=8$ for day $2, n=6$ for day $3, n=4$ for day 4 , and $n=2$ for day 7 . $\mathrm{TCID}_{50}$, median tissue culture infections dose. 


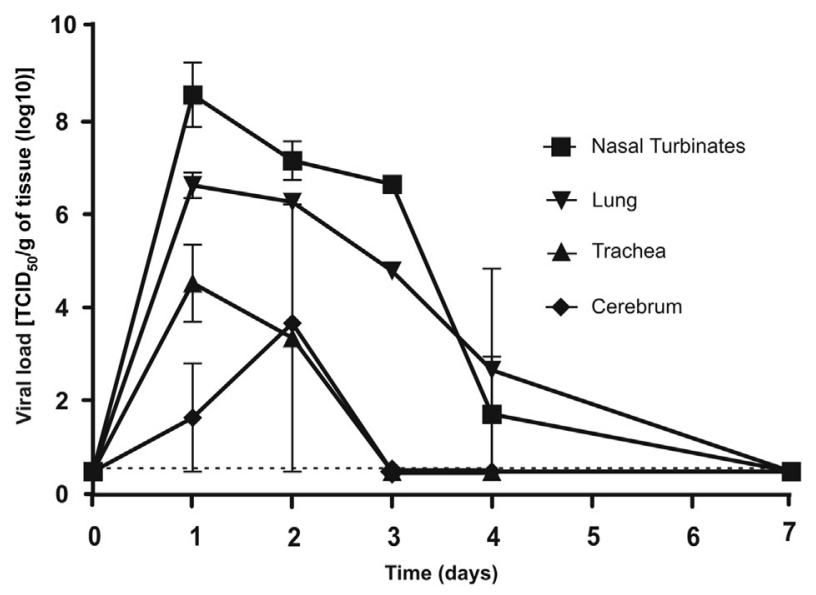

Figure 2 Tissue viral load after intratracheal inoculation with influenza $\mathrm{A}(\mathrm{H} 1 \mathrm{~N} 1) p d m 09$ virus. Dashed line indicates limit of detection $<0.75$ $\mathrm{TCID}_{50} / \mathrm{g}$ (10log). $n=2$ per time point. $\mathrm{TCID}_{50}$, median tissue culture infections dose.

group additionally displayed ruffled fur and slightly decreased activity. From day 0 to day 1 guinea pigs displayed modest mean weight loss of $5.4 \%$ and $4.4 \%$ for the i.t.- and i.n.-inoculated guinea pigs, respectively.

\section{Virus Replication}

Two days after inoculation, virus titers in the nasal swabs reached a peak in all guinea pigs. By day 7 after inoculation, infectious virus was no longer detectable in the nasal swabs $\left[<0.75 \mathrm{TCID}_{50} / \mathrm{mL}(10 \mathrm{log})\right]$. After i.n. inoculation, higher virus titers were observed in nasal secretions than after i.t. inoculation [peak titers, $4.2 \pm \mathrm{SD} 1.2$ and $2.5 \pm \mathrm{SD} 1.5$ $\mathrm{TCID}_{50} / \mathrm{mL}$ (10log)] (Figure 1). Pharyngeal swabs were intermittently positive for all guinea pigs but did not show a clear pattern of virus excretion (data not shown). Infectious virus was not detected in any of the rectal swabs.

In all guinea pigs that were inoculated via the i.n. route, infectious virus was detected in the nasal turbinates on day 4 after inoculation $(n=6)$ with virus titers ranging from 3.0 to $5.9 \mathrm{TCID}_{50} / \mathrm{g}(10 \mathrm{log})\left[\right.$ means $\pm \mathrm{SD}, 4.1 \pm 1.2 \mathrm{TCID}_{50} / \mathrm{g}$ $(10 \log )]$. At this time point, virus could not be isolated from remaining tissues, with the exception of the cerebrum of one of six guinea pigs, with a titer of $2.5 \mathrm{TCID}_{50} / \mathrm{g}(10 \mathrm{log})$ (data not shown).

To investigate the kinetics of virus replication in various tissues after i.t. inoculation, 10 strain 2 guinea pigs were inoculated, and at various time points after inoculation 2 guinea pigs were sacrificed. On the basis of the results obtained after i.n. inoculation, autopsies at earlier time points (days 1, 2, and 3) were included. Widespread virus replication was observed (Figure 2) with infectious virus detected in the nasal turbinates on day 1, 2, $3(n=2$ of 2 guinea pigs), and 4 after inoculation $(n=1$ of 2$)$ [means \pm SD peak virus titer on day $\left.1,8.1 \pm 1.7 \mathrm{TCID}_{50} / \mathrm{g}(10 \log )\right]$; trachea on day $1(n=2$ of 2$)$ and 2 after inoculation $(n=1$ of 2) [means \pm SD peak virus titer on day $1,4.6 \pm 0.6$
$\left.\mathrm{TCID}_{50} / \mathrm{g}(10 \log )\right]$; lung on day $1,2,3(n=2$ of 2$)$, and 4 $(n=1$ of 2 ) [means \pm SD peak virus titer on day $1,6.7 \pm$ $\left.0.4 \mathrm{TCID}_{50} / \mathrm{g}(10 \mathrm{log})\right]$; and cerebrum on day $1(n=1$ of 2$)$ and $2(n=2$ of 2$)$ after inoculation [means \pm SD peak virus titer on day $\left.2,3.7 \pm 0.1 \mathrm{TCID}_{50} / \mathrm{g}(10 \log )\right]$.

\section{Macroscopic and Microscopic Findings}

Macroscopically, lungs of i.n.-inoculated guinea pigs showed multifocal small areas of consolidation affecting $<15 \%$ of lung tissue. At day 4 and 7 after inoculation, a moderate quantity of mucopurulent exudate was present in the nasal cavity, and the mucosa showed diffuse hyperemia.

Lungs of i.t.-inoculated guinea pigs were visibly more severely affected, with $\leq 30 \%$ of tissue showing randomly distributed, well-demarcated, firm, dark red foci in pulmonary parenchyma (Figure 3). Within the nasal cavity, especially posteriorly, a large quantity of mucopurulent exudate was present from day 2 to day 7 after inoculation, and the mucosa was diffusely hyperemic. Other tissues showed no notable macroscopic changes.

Histologic evaluation of tissues from i.n.-inoculated guinea pigs revealed moderate inflammation of the nasal epithelium and mild inflammation in the remaining respiratory tract, with an inflammatory infiltrate composed predominantly of polymorphonuclear cells morphologically consistent with eosinophils, fewer heterophils and, especially in the pulmonary parenchyma, mononuclear cells morphologically consistent with macrophages.

On days 4 and 7 after inoculation, all respiratory tract tissues and particularly alveoli from i.t.-inoculated guinea pigs showed more widespread and more severe inflammation of the alveolar walls and respiratory epithelium (and, where present, the submucosa) than alveoli of i.n.-inoculated guinea pigs (Figure 4). After i.t. inoculation, the extent of acute inflammation was most marked at approximately day 2 and 3 after inoculation (Figure 5) and infrequently accompanied by intraalveolar edema and deposition of fibrin. Pyknosis, karyorrhexis, and karyolysis (necrosis), flattening (attenuation), and/ or loss of cilia of epithelial cells of nasal cavity, bronchi(oli), and alveoli were more frequently observed with this route of
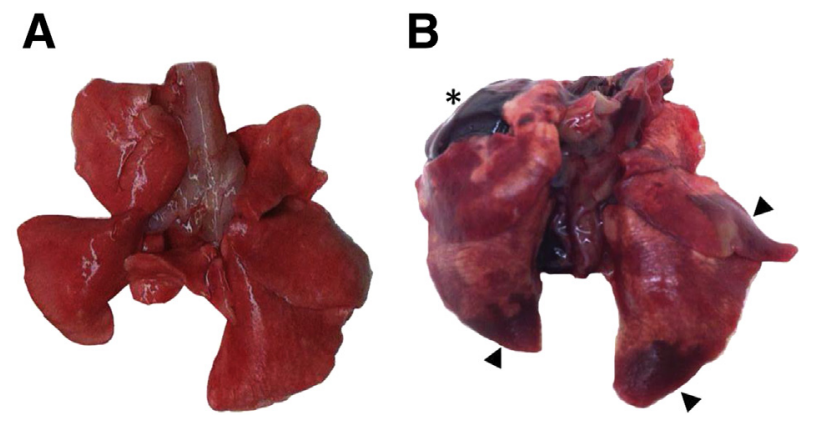

Figure 3 Macroscopic image of guinea pig lung. A: Normal guinea pig lung. B: Guinea pig lung at 2 days after intratracheal inoculation; arrowheads indicate multiple areas of consolidation, and asterisk shows heart (not present in control). 


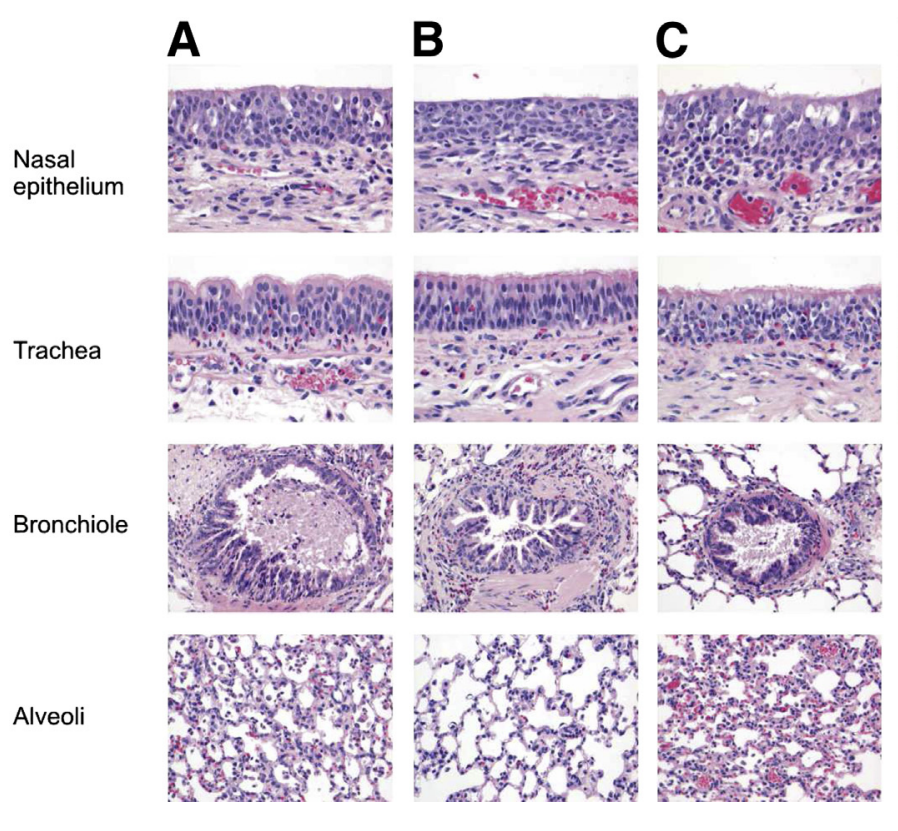

inoculation than with i.n. inoculation. Necrotic debris frequently accumulated in alveolar and airway lumina and on nasal epithelium. Some type II pneumocyte hyperplasia was seen in the alveoli by day 7 after inoculation (regeneration).

Other tissues examined, including the cerebrum, showed no notable microscopic changes apart from one i.n.-inoculated guinea pig that showed perivascular cuffing with mononuclear cells (approximately 5 cells thick) in the olfactory lobe at day 7 after inoculation. However, this guinea pig showed no detectable virus titers or antigen positivity by IHC analysis in the brain.

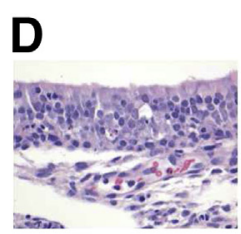

Figure 4 Histopathology (H\&E) of nasal epithelium and trachea and bronchiole and alveoli. A: Intratracheal inoculation 4 days after inoculation. B: Intranasal inoculation 4 days after infection. C: Intratracheal inoculation 7 days after infection. D: Intranasal inoculation 7 days after infection. Inflammatory changes are more pronounced after intratracheal inoculation. Original magnification: $\times 40(\mathbf{A}-\mathbf{D}$, epithelium and trachea); $\times 20(\mathbf{A}-\mathbf{D}$, bronchiole and alveoli). $H \& E$, hematoxylin and eosin.

\section{IHC Findings}

After i.n. inoculation, moderate numbers of viral antigenpositive cells were detected in the nasal epithelium at day 4 and, to a lesser extent, day 7 after inoculation. Virus-infected cells were not detected in any other (respiratory) tissues (data not shown).

After i.t. inoculation, moderate numbers of virus-infected (epithelial) cells were detected by IHC analysis in all respiratory tissues (Figure 6). Nasal epithelium showed a peak in positivity at day 2 after inoculation with a steady decline on

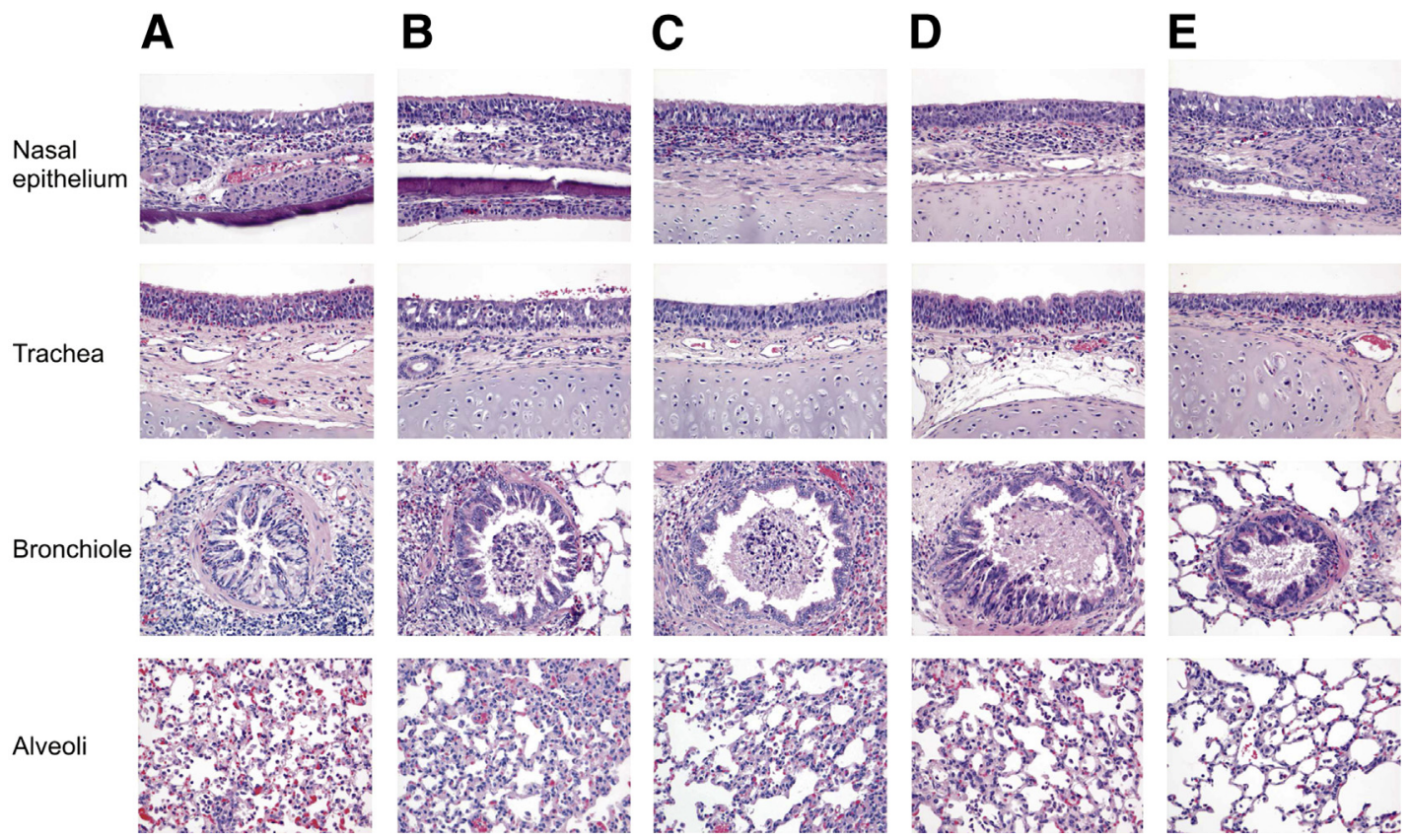

Figure 5 Histopathology (H\&E) of respiratory tissues after intratracheal inoculation at days 1 (A), 2 (B), 3 (C), 4 (D), and 7 (E) after inoculation. Acute inflammatory changes and necrosis are most prominent at days 2 and 3 after inoculation. Original magnification, $\times 20$. H\&E, hematoxylin and eosin. 


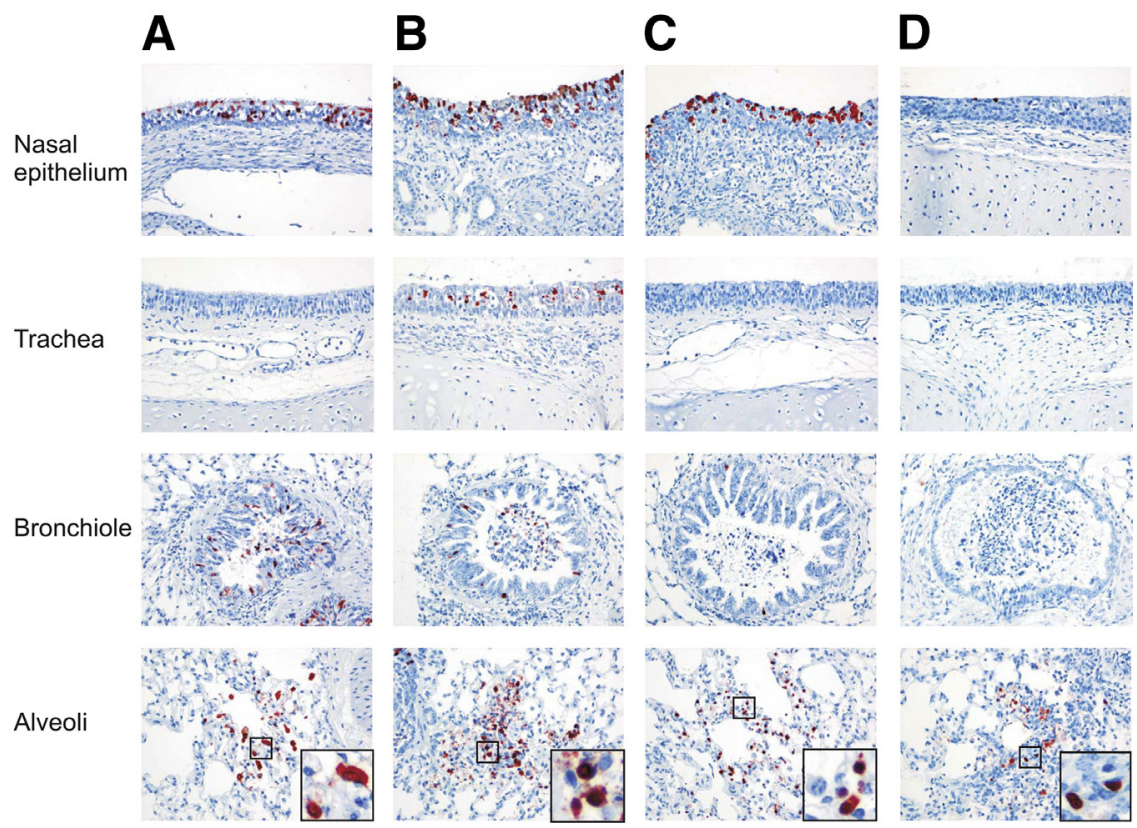

Figure 6 Immunohistochemistry of respiratory tract tissues after intratracheal inoculation. One day (A), 2 days (B), 3 days (C), and 4 days (D) after infection. Intranasal inoculation resulted in infection of cells in the nasal epithelium only (results not shown). Insets show boxed areas at higher magnification. Original magnification, $\times 20$ (main images) $; \times 80$ (insets). day 3 and 4 and was negative by day 7. Trachea was antigen positive on day 2 after inoculation only. Bronchi(oli) showed peak positivity on day 1 , followed by a steady decline on day 2,3 , and 4 , and by day 7 antigen was no longer detectable. Antigen in alveoli also peaked at day 2 after inoculation and thereafter declined steadily with only a few cells positive by day 7. The number of virus-infected cells in the respiratory tract therefore roughly correlated with the virus titers that were measured in the respective tissues (Figure 2).

Cell types that showed intranuclear antigen were epithelial cells of the upper respiratory tract and airways and type II pneumocytes and alveolar macrophages in the pulmonary parenchyma (Figure 6). These cells were identified on the basis of their location and structure. Other tissues inspected, including the cerebrum, showed no virus antigen positivity.

Major basic protein antibody (clone BMK-13) was used to definitively distinguish eosinophils from heterophils. In respiratory tissues, the predominant polymorphonuclear cell type present was confirmed to be eosinophils (Figure 7).

\section{Discussion}

In the present study we investigated virus replication and pathogenesis of infection with $\mathrm{A}(\mathrm{H} 1 \mathrm{~N} 1) \mathrm{pdm} 09$ influenza virus in strain 2 isogenic guinea pigs after i.n. or i.t. inoculation. For both inoculation routes, virus excretion from the nose peaked at approximately day 2 after inoculation, although after i.n. inoculation higher virus titers were observed in the nose swabs than after i.t. inoculation. After i.n. inoculation, virus replication in the respiratory tract was restricted to the nasal epithelium. In one guinea pig virus was also detectable in the cerebrum on day 4 after inoculation. In contrast, i.t. inoculation appeared to result in more widespread replication. On days 1, 2, 3, and 4 after inoculation, infectious virus was not only detected in the nasal epithelium but also in the lungs and on days 1 and 2 in the trachea and the cerebrum. Because of the limited breeding capacity at present and restrictions imposed by the ethical committee, it was not possible to test virus replication in the respective organs at earlier time points after i.n. inoculation. It therefore remains unclear whether the virus replicated in the respective respiratory tissues before day 4 after inoculation. Previous studies do not consistently report spread of influenza viruses to the lower respiratory tract after i.n. inoculation, but the histologic lesions observed in the lungs of the i.n.-inoculated guinea pigs were consistent with those previously described for infection with influenza viruses of other subtypes. ${ }^{24,27-29}$ However, in the present study we could not confirm viral replication (by detection of infectious virus or infected cells) in tissues other than the nasal epithelium after i.n. inoculation.

For i.t. inoculation, the results of IHC analysis (Figures 6 and 7) corresponded with the virus titers in the respective

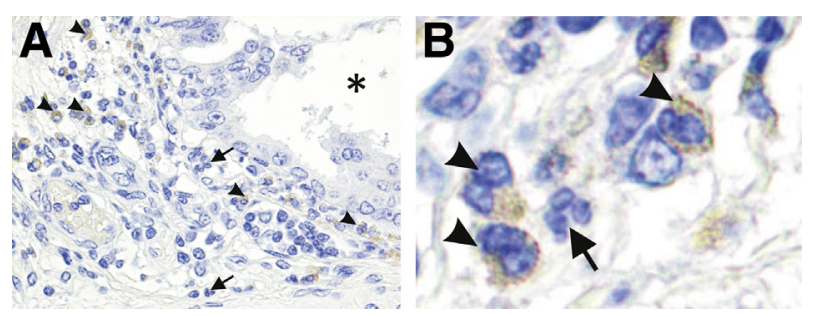

Figure 7 A: Immunohistochemistry of inflammatory infiltrate surrounding a bronchus (asterisk) at day 2 after intratracheal inoculation. B: Cells at higher magnification. Arrowheads show eosinophils with granules that stain positive (brown) with major basic protein antibody (BMK-13); arrows show heterophils. Original magnification: $\times 20(\mathbf{A}) ; \times 80(\mathbf{B})$. 
tissues (Figure 2), confirming the presence of virus in epithelial cells of the nasal turbinates, trachea, bronchi(oli), alveoli and in pulmonary macrophages between day 1 and 4 after inoculation. Low virus titers were detected in the cerebrum at day 1 ( $n=1$ of 2$)$ and $2(n=2$ of 2$)$; however, no antigen-positive cells were detected by IHC analysis. No histologic changes were noted in the cerebrum of any of the guinea pigs apart from one i.n.-inoculated guinea pig that showed minimal histologic changes in the olfactory lobe at day 7 after inoculation but did not show detectable viral titers or viral antigen positivity. The relevance of this finding and the potential importance of the olfactory route of entry into the guinea pig central nervous system therefore remains unknown.

Interestingly, i.t. inoculation resulted in effective spread of replicating virus to both the upper and lower respiratory tracts. In ferrets, i.t. inoculation with 2009 pandemic $\mathrm{A}(\mathrm{H} 1 \mathrm{~N} 1)$ virus typically results in viral replication predominantly in the lower respiratory tract. ${ }^{10,30,31}$ If i.t. inoculation becomes the preferred route of inoculation for guinea pigs, this is especially relevant for future transmission studies. For both routes of inoculation, the trachea appeared to be relatively spared. Histologic lesions in the trachea were mild, replicating virus was only demonstrated in 3 of 10 i.t.-inoculated guinea pigs, and IHC analysis was positive in only 1 guinea pig. This may be explained by the relatively small number of cells available for infection in the trachea compared with the lower respiratory tract.

Overall, histologic lesions corresponded generally with those reported in the few pathogenesis studies previously conducted in guinea pigs ${ }^{24,27-29}$ and consisted of rhinitis, tracheitis, bronch(iol)itis, and alveolitis with mild (i.n. inoculation) and moderate (i.t. inoculation) infiltrates consisting of numerous eosinophils, fewer heterophils, and, in the lower respiratory tract, macrophages. Epithelial attenuation and necrosis were noted multifocally throughout the respiratory tract and were more marked especially in the lower respiratory tract after i.t. inoculation.

In two of the previous guinea pig influenza studies that report on viral pathogenesis, the investigators comment on the presence of neutrophils. ${ }^{28,29}$ Guinea pig polymorphonuclear cells that correspond to neutrophils are more correctly referred to as heterophils. ${ }^{32}$ Guinea pig heterophils and eosinophils are easily confused at first glance because of the presence of a segmented nucleus and abundant eosinophilic cytoplasmic granules in both cell types. They can be distinguished to some extent on the basis of nuclear and granule structure; heterophils are slightly smaller and have a more segmented nucleus and fewer and smaller cytoplasmic granules than eosinophils. However, when an infiltrate is composed of only one of these two cell types, these relative distinguishing features are less useful, and so additional methods may become of interest, especially considering that the functional differences of these cell types parallel those in other species. Major basic protein is a constituent of eosinophil granules, ${ }^{33}$ and, here, we show that antibody directed against this protein (clone BMK-13) can be used to definitively distinguish eosinophils from heterophils. To our knowledge, no existing literature used this method in guinea pigs, and we hereby recommend its use in cases in which accurate description of the inflammatory infiltrate is of importance. With the use of major basic protein antibody immunohistochemistry, we found that eosinophils were the predominant polymorphonuclear cell type in airway infiltrates of infected guinea pigs. Tang et $\mathrm{al}^{28}$ also report the presence of eosinophils in the airways of infected animals and speculate that this finding is suggestive of airway hypersensitivity. Inbred guinea pigs were previously shown to mount a delayed hypersensitivity response to influenza $\mathrm{A}$ infections with the use of nasal washes and peritoneal exudates, ${ }^{34}$ a finding that would be consistent with these observations. However, the exact significance of this finding in the pathologic response to influenza remains to be elucidated.

In summary, we have explored, for the first time, infection of isogenic strain 2 guinea pigs with pandemic H1N1 influenza virus as an influenza animal model. The i.t. and i.n. routes of inoculation were compared, and, for both procedures, productive infection of the upper respiratory tract was achieved, with a peak in viral replication at approximately day 2 after inoculation. The i.t. inoculation appeared to result in more widespread viral replication and associated histologic lesions in both the upper and lower respiratory tracts, whereas after i.n. inoculation hisotpathologic changes were more restricted to the nasal cavity. Thus, compared with i.n. inoculation, the pathogenesis of infection of guinea pigs after i.t. inoculation may mimic viral pneumonia in humans more closely. However, i.t. administration is a technically more demanding procedure. In addition to these findings, we presented a novel way to accurately distinguish heterophils from eosinophils in guinea pig formalin-fixed, paraffinembedded tissue sections by using major basic protein antibody (BMK-13).

The use of this particular inbred strain of guinea pigs seems a promising model for human infections with influenza virus. Furthermore, it would allow investigation of virus-specific cellular immunity and identification of immune correlates of cross-protection against influenza virus infection by adoptive transfer of selected lymphocyte subsets. Because guinea pigs transmit influenza virus efficiently, the model also allows studying the effect of immunity on virus transmission.

\section{References}

1. Garten RJ, Davis CT, Russell CA, Shu B, Lindstrom S, Balish A, et al: Antigenic and genetic characteristics of swine-origin 2009 A(H1N1) influenza viruses circulating in humans. Science 2009, 325:197-201

2. Novel Swine-Origin Influenza $\mathrm{A}(\mathrm{H} 1 \mathrm{~N} 1)$ Virus Investigation Team, Dawood FS, Jain S, Finelli L, Shaw MW, Lindstrom S, Garten RJ, Gubareva LV, Xu X, Bridges CB, Uyeki TM: Emergence of a novel swine-origin influenza A (H1N1) virus in humans. N Engl J Med 2009, 360:2605-2615

3. Neumann G, Chen H, Gao GF, Shu Y, Kawaoka Y: H5N1 influenza viruses: outbreaks and biological properties. Cell Res 2010, 20:51-61 
4. Gong Z, Lv H, Ding H, Han J, Sun J, Chai C, Cai J, Yu Z, Chen E: Epidemiology of the avian influenza A (H7N9) outbreak in Zhejiang Province, China. BMC Infect Dis 2014, 14:244

5. Liu J, Xiao H, Wu Y, Liu D, Qi X, Shi Y, Gao GF: H7N9: a low pathogenic avian influenza A virus infecting humans. Curr Opin Virol 2014, 5:91-97

6. Bodewes R, Rimmelzwaan GF, Osterhaus AD: Animal models for the preclinical evaluation of candidate influenza vaccines. Expert Rev Vaccines 2010, 9:59-72

7. Thangavel RR, Bouvier NM: Animal models for influenza virus pathogenesis, transmission, and immunology. J Immunol Methods 2014, 4:60-79

8. Bouvier NM, Lowen AC: Animal models for influenza virus pathogenesis and transmission. Viruses 2010, 2:1530-1563

9. Kroeze EJ, Kuiken T, Osterhaus AD: Animal models. Methods Mol Biol 2012, 865:127-146

10. van den Brand JM, Haagmans BL, van Riel D, Osterhaus AD, Kuiken T: The pathology and pathogenesis of experimental severe acute respiratory syndrome and influenza in animal models. J Comp Pathol 2014, 151:83-112

11. Jayaraman A, Chandrasekaran A, Viswanathan K, Raman R, Fox JG, Sasisekharan R: Decoding the distribution of glycan receptors for human-adapted influenza A viruses in ferret respiratory tract. PLoS One 2012, 7:e27517

12. Bodewes R, Kreijtz JH, van Amerongen G, Fouchier RA, Osterhaus AD, Rimmelzwaan GF, Kuiken T: Pathogenesis of Influenza $\mathrm{A} / \mathrm{H} 5 \mathrm{~N} 1$ virus infection in ferrets differs between intranasal and intratracheal routes of inoculation. Am J Pathol 2011, 179:30-36

13. Lowen AC, Mubareka S, Tumpey TM, Garcia-Sastre A, Palese P: The guinea pig as a transmission model for human influenza viruses. Proc Natl Acad Sci U S A 2006, 103:9988-9992

14. Moncla LH, Ross TM, Dinis JM, Weinfurter JT, Mortimer TD, Schultz-Darken N, Brunner K, Capuano SV 3rd, Boettcher C, Post J, Johnson M, Bloom CE, Weiler AM, Friedrich TC: A novel nonhuman primate model for influenza transmission. PLoS One 2013, 8:e78750

15. Kreijtz JH, Fouchier RA, Rimmelzwaan GF: Immune responses to influenza virus infection. Virus Res 2011, 162:19-30

16. Hillaire ML, van Trierum SE, Kreijtz JH, Bodewes R, GeelhoedMieras MM, Nieuwkoop NJ, Fouchier RA, Kuiken T, Osterhaus AD, Rimmelzwaan GF: Cross-protective immunity against influenza pH1N1 2009 viruses induced by seasonal influenza A (H3N2) virus is mediated by virus-specific T-cells. J Gen Virol 2011, 92:2339-2349

17. Lipscomb MF, Lyons CR, O'Hara RM Jr, Stein-Streilein J: The antigen-induced selective recruitment of specific $\mathrm{T}$ lymphocytes to the lung. J Immunol 1982, 128:111-115

18. Phair JP, Kauffman CA, Jennings R, Potter CW: Influenza virus infection of the guinea pig: immune response and resistance. Med Microbiol Immunol 1979, 165:241-254

19. Schafer H, Burger R: Tools for cellular immunology and vaccine research the in the guinea pig: monoclonal antibodies to cell surface antigens and cell lines. Vaccine 2012, 30:5804-5811

20. Mizutani N, Inui $\mathrm{S}$, Yoshino $\mathrm{S}$, Nabe $\mathrm{T}$ : Intratracheal sensitization/challenge-induced biphasic asthmatic response and airway hyperresponsiveness in guinea pigs. Biol Pharm Bull 2010, 33:1949-1952

21. Lipscomb MF, Yeakel-Houlihan D, Lyons CR, Gleason RR, SteinStreilein J: Persistence of influenza as an immunogen in pulmonary antigen-presenting cells. Infect Immun 1983, 42:965-972

22. Edelstein PH: The Guinea pig model of legionnaires' disease. Methods Mol Biol 2013, 954:521-540

23. Steel J, Staeheli P, Mubareka S, Garcia-Sastre A, Palese P, Lowen AC: Transmission of pandemic H1N1 influenza virus and impact of prior exposure to seasonal strains or interferon treatment. J Virol 2010, 84: $21-26$

24. Azoulay-Dupuis E, Lambre CR, Soler P, Moreau J, Thibon M: Lung alterations in guinea-pigs infected with influenza virus. J Comp Pathol 1984, 94:273-283

25. Rimmelzwaan GF, Baars M, Claas EC, Osterhaus AD: Comparison of RNA hybridization, hemagglutination assay, titration of infectious virus and immunofluorescence as methods for monitoring influenza virus replication in vitro. J Virol Methods 1998, 74:57-66

26. van den Brand JM, Stittelaar KJ, van Amerongen G, Reperant L, de Waal L, Osterhaus AD, Kuiken T: Comparison of temporal and spatial dynamics of seasonal $\mathrm{H} 3 \mathrm{~N} 2$, pandemic $\mathrm{H} 1 \mathrm{~N} 1$ and highly pathogenic avian influenza H5N1 virus infections in ferrets. PLoS One 2012, 7:e42343

27. Van Hoeven N, Belser JA, Szretter KJ, Zeng H, Staeheli P, Swayne DE, Katz JM, Tumpey TM: Pathogenesis of 1918 pandemic and $\mathrm{H} 5 \mathrm{~N} 1$ influenza virus infections in a guinea pig model: antiviral potential of exogenous alpha interferon to reduce virus shedding. J Virol 2009, 83:2851-2861

28. Tang X, Chong KT: Histopathology and growth kinetics of influenza viruses (H1N1 and H3N2) in the upper and lower airways of guinea pigs. J Gen Virol 2009, 90:386-391

29. Kwon YK, Lipatov AS, Swayne DE: Bronchointerstitial pneumonia in guinea pigs following inoculation with $\mathrm{H} 5 \mathrm{~N} 1$ high pathogenicity avian influenza virus. Vet Pathol 2009, 46:138-141

30. Kreijtz JH, Suzer Y, Bodewes R, Schwantes A, van Amerongen G, Verburgh RJ, de Mutsert G, van den Brand J, van Trierum SE, Kuiken T, Fouchier RA, Osterhaus AD, Sutter G, Rimmelzwaan GF: Evaluation of a modified vaccinia virus Ankara (MVA)-based candidate pandemic influenza A/H1N1 vaccine in the ferret model. J Gen Virol 2010, 91:2745-2752

31. van den Brand JM, Stittelaar KJ, van Amerongen G, Rimmelzwaan GF, Simon J, de Wit E, Munster V, Bestebroer T, Fouchier RA, Kuiken T, Osterhaus AD: Severity of pneumonia due to new H1N1 influenza virus in ferrets is intermediate between that due to seasonal H1N1 virus and highly pathogenic avian influenza H5N1 virus. J Infect Dis 2010, 201:993-999

32. Brederoo P, Daems WT: The ultrastructure of guinea pig heterophil granulocytes and the heterogeneity of the granules. Cell Tissue Res 1978, 194:183-205

33. Gleich GJ, Loegering DA, Maldonado JE: Identification of a major basic protein in guinea pig eosinophil granules. J Exp Med 1973, 137 : 1459-1471

34. Wetherbee RE: Induction of systemic delayed hypersensitivity during experimental viral infection of the respiratory tract with a myxovirus or paramyxovirus. J Immunol 1973, 111:157-163 